\title{
Design and Study of the Observation Optics for the Thomson Scattering Planned at Wendelstein 7-X
}

\author{
J. Cantarini, J.P. Knauer and E. Pasch \\ Max-Planck-Institut für Plasmaphysik EURATOM Association \\ Wendelsteinstr.1, 17491 Greifswald, Germany
}

\begin{abstract}
The main aim of the Thomson scattering system is the measurement of electron temperature and density profiles with high time and spatial resolution. To cover the whole laser beam line $(1.6 \mathrm{~m})$ through the plasma cross section, two ports are provided for the observation optics, which image the scattering volumes (each with $28 \mathrm{~mm}$ length and $9 \mathrm{~mm}$ diameter) onto fiber bundles. The observation optics are important components of the diagnostic set-up, because their imaging properties determine the spectral and spatial resolution of the whole system. Therefore the design of the optics must be optimized according to the geometrical constrains of the observation ports in terms of position and dimensions. To optimize this optical engineering, the commercial ZEMAX program is used. The composition of the optical system is elaborated to minimize losses of collected light with wavelength from $700 \mathrm{~nm}$ up to $1064 \mathrm{~nm}$. Environmental criteria (e.g. neutrons, ECR plasma heating and temperature) will be considered choosing optical materials. First results of calculations will be presented.
\end{abstract}

Keywords: Thomson scattering, Laser plasma diagnostics, Collection optics, Scheimpflug imaging

\section{INTRODUCTION}

The goal of the Thomson scattering diagnostic at Wendelstein 7- X stellerator is to provide reliable electron temperature and density profiles in both core and edge regions of the plasma. The system has to measure complete Te and ne profiles with spatial resolution of $2 \mathrm{~cm}$ in the range $20 \mathrm{eV}<\mathrm{T}_{\mathrm{e}}<10 \mathrm{keV}$ and $5 \cdot 10^{18} \mathrm{~m}^{-3}<\mathrm{n}_{\mathrm{e}}<5 \cdot 10^{20} \mathrm{~m}^{-3}$. To reach this goal, three collection optical systems should be designed for the W7-X Thomson scattering diagnostic to optimize the coverage view and the optical throughput. In this article, we describe first design studies of one of the collection optic covering the core and the edge of the half diameter of the plasma.

\section{GENERAL DESCRIPTION OF THE SYSTEM}

\section{Description of the Optical Device}

In order to achieve the required performance, the Thomson scattering systems employ a set of 5 Nd:YAG laser beams. The system, which is allocated in port AEM 31 and AEN 31, covers a line of sight of $1.6 \mathrm{~m}$, as shown in Figure 1. In this paper, we present one of the set of collection optics allocated in AEM 31 port. The other two systems will be allocated in AEN 31 port. The scattered light is imaged onto optical fiber bundles, located inside the port, which transmit the light to the spectra analyzing system (polychromator). The collection optics is situated inside the port and in air, protected by a circular quartz window of $220 \mathrm{~mm}$ diameter and $20 \mathrm{~mm}$ thickness, which is mounted in front of the lenses as shown in figure 2. This window is used as a vacuum seal. A shutter mechanism is installed in front of the window. Lasers and polychromators are located in a diagnostic room, outside the torus hall.

Inside the port where optics are located, we have to consider space between the port and the internal rails of optics because of thermal movement of the ports. This margin space is $2 \mathrm{~cm}$. During the optical design optimization, we also consider the dimension of the structure of internal rails which support lenses. 


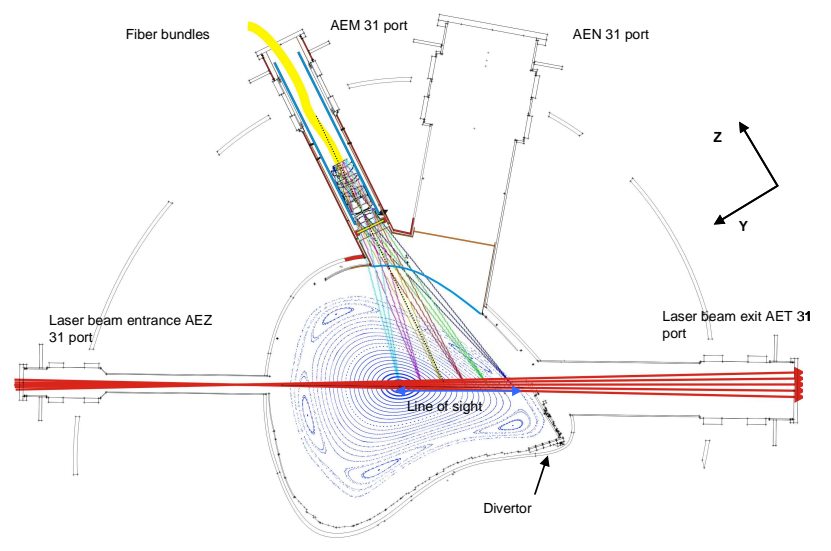

FIGURE 1. Optical system in AEM 31. A laser fan with five Nd: YAG laser beams is shown.

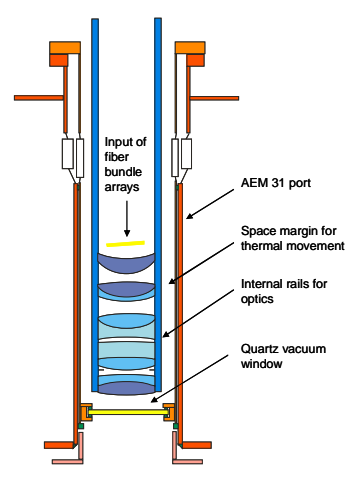

FIGURE 2. Collection optics allocated inside the AEM 31 port.

\section{Environmental Constraints}

The first constraint is carbon deposition on the vacuum window. During diagnostics measurement, material deposition can appear and reduce the transmission of the window. This deposition is a recurrent constraint for observation optics in fusion devices. To resolve this issue, a shutter is set to protect the window from material deposition between measurements. Nevertheless after many experiments, the carbon deposit on the window can reduce the visibility of our optics. In order to avoid lower transmission by carbon deposit layer, we envisage the possibility to heat the window in order to remove the carbon deposition and restore the good transmission of the optical set ${ }^{1}$. The second constraint is the neutron radiation inside the port. In the port we could reach a neutron flux up to $7 \cdot 10^{9} \mathrm{~cm}^{-2} \mathrm{~s}^{-1}$ during discharge knowing that the allowed limit is fixed to a neutron flux of $3 \cdot 10^{19} \mathrm{~cm}^{-2}$ per year. We use neutron radiation resistant materials for our system. The third constraint is the magnetic field, which can reach up to $3 \mathrm{~T}$ on the plasma axis. Due to that, we won't use any magnetic materials $\left(\mu_{\mathrm{r}}<1,05\right)$ for our observation system . The fourth constraint is the temperature caused by plasma radiation and ECRH stray radiation at Thomson scattering port which is roughly $30 \mathrm{~kW} / \mathrm{m}^{2}$. Therefore a cooling system inside the port is foreseen.

\section{OPTICAL DESIGN}

The collection optics for Thomson scattering system is shown in figure 4. The system of 9 lenses is designed to achieve a full field of view of $25,5^{\circ}$. In order to have good collectivity, optics have a diameter of $170 \mathrm{~mm}$. The image $f$-number is 1,37 for a focal length of $193 \mathrm{~mm}$. The image numerical aperture is 0,33 . The scattered light is collected onto optical fiber bundle arrays and relayed a long distance to the polychromator, which are located at the Thomson diagnostic room. The shape of each fiber bundle input is designed to be rectangular with a fixed surface (7 $\mathrm{mm}^{2}$ ) which corresponds to the circular shape $(3 \mathrm{~mm}$ diameter) of each fiber bundle output according to the 
polycromator design ${ }^{2}$. The optics are optimized for spectral range from $700 \mathrm{~nm}$ up to $1064 \mathrm{~nm}$ according to the Thomson scattering spectra of electron temperature up to $10 \mathrm{keV}$.

\section{Optical Parameters}

In order to provide a good edge and core view of Thomson scattering laser with optical performance expected as a demagnification greater than 6:1 and an image numerical aperture of 0,37 determined by our fibers, we started focusing on field of view, spot size and spectral range parameters of the system. The first design was performed on spot size by optimizing curvature, thickness and glass types. Target values like magnification, image numerical and field of view were considered. We performed the collection optics on the AEM 31 port in order to cover the line of sight of $820 \mathrm{~mm}$ from the edge to the core of the plasma, which is tilted of $25,8^{\circ}$ to the optical axis of the collection optics.

\section{Scheimpflug Condition}

According to the geometrical constraints of the observation port, the line of sight is not perpendicular to the optical axis of our collection optic in AEM31 port. Therefore the magnification along the image plane will be nonuniform. To know and control the magnification of our system, we have to consider the Scheimpflug imaging. The Scheimpflug condition says that if the object is tilted from the lens optical axis than the image plane will be also tilted. The Scheimpflug condition indicates how to find the image plane tilt produced by an object plane tilted with respect of the optical axis. The way to construct the Scheimpflug condition is described by the figure 3 . The object plane $(\mathrm{AB})$ must intersect the object principal plane $\mathrm{P}$ defining the point of intersection of the image plane (A'B') with the image principal plane P'. If this condition is satisfied, the image is sharp. We want to image each volume of scattered light to one specific fiber bundle with fixed input. Our fiber bundle array associated with the image plane must be located in the sharp area and thus our system must satisfy Scheimpflug condition.

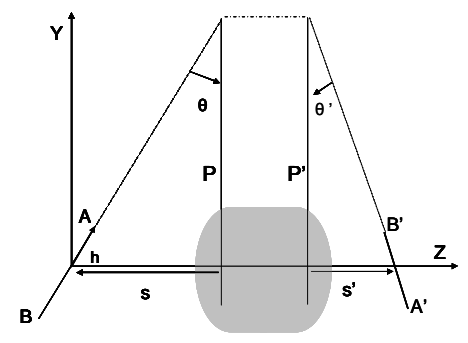

FIGURE 3. Scheimpflug condition for a thick optical system.

In this case the magnifications depend on $\theta$ and the object height $\mathrm{h}$. Thus, the differential local magnification along the image plane can be described by the following equations ${ }^{3}$, where $s, s^{\prime}, \theta, \theta$ ' are described in figure 3 :

$$
\mathrm{M}_{\mathrm{x}}=\left[\frac{\mathrm{s} \mathrm{m}_{0}}{\mathrm{~s}+\mathrm{h}\left(1-\mathrm{m}_{0}\right) \sin \theta}\right], \mathrm{M}_{y}=\left[\frac{\mathrm{s} \mathrm{m}_{0}}{\mathrm{~s}+\mathrm{h}\left(1-\mathrm{m}_{0}\right) \sin \theta}\right]^{2}\left[\frac{\sin \theta}{\sin \theta^{\prime}}\right], \mathrm{m}_{0}=\frac{s^{\prime}}{\mathrm{s}}
$$

Correspondingly there is a keystone distortion in the Scheimpflug imaging. This geometrical distortion results in a trapezoidal image of a nominally rectangular object ${ }^{4}$. This shape deformation must be predicted in order to design the appropriate dimension of the fiber bundle inputs. This oblique imaging case doesn't need specific lenses. It is shown that non oblique imaging lens systems which show good imaging properties can give good results in oblique imaging if they satisfy Scheimpflug condition ${ }^{5}$.

\section{Results}

The system is able to collect the scattered light via a quartz window by a set of BK7, LAK9 and SF6 neutron resistant glasses constituted by a 4 achromats and a meniscus has been added to enhance the image numerical aperture. The ray tracing of this set of lens is described in the following figure 4. 


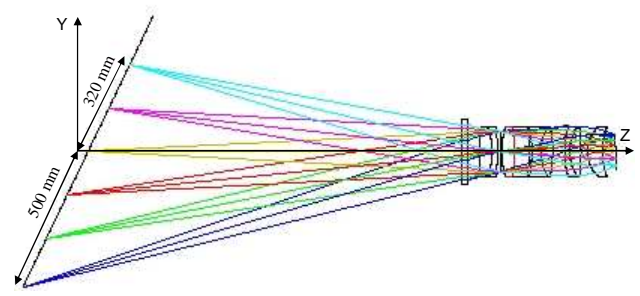

FIGURE 4. ZEMAX program ray tracing.

When we start the design of the first set of collection optics, we wanted to reach a demagnification better than 6:1. Considering the scattering geometry and optical design, we could cover half of the line of sight of the plasma with one optical system reaching a non-uniform demagnification from 11:1 for the edge and to 6:1 for the core. The Thomson scattering collection optics presents a vignetting less than $10 \%$ from the optical axis up to $423 \mathrm{~mm}$. The distance of the optical axis to the plasma center is $320 \mathrm{~mm}$. This line of sight covers the plasma up to the separatrix. Beyond the separatrix, in the island region, vignetting comes important and reaches $56 \%$ at the extremity of the line of sight, as shown in figure 5. The edge of the plasma can be observed.

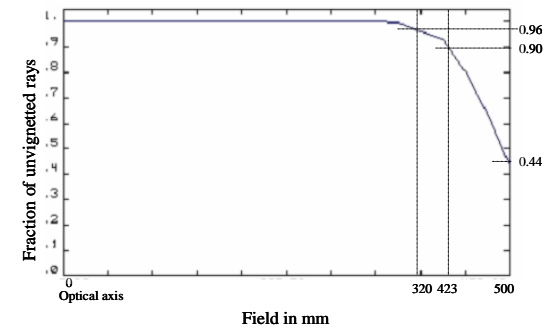

FIGURE 5. Vignetting diagram.

\section{FUTURE WORK}

On one hand, in order to improve the optimization, we want to focus our ZEMAX calculations on reducing spot size and chromatic aberrations according to the spatial resolution expected. On the second hand, we will design the optics for the AEN 31 port to cover the other half of the plasma and study the possibilities to use one or two sets of the optics due to the geometry constraints of the AEN 31 port. Than fiber bundles will be designed.

\section{ACKNOWLEDGMENTS}

The authors want to thank Mr. B. Sitkowski for his helpful technical support.

\section{REFERENCES}

1. H-J. Hartfuss, R. König, A. Werner, Diagnostics for steady state plasmas, Plasma Physics and Controlled Fusion 48, 2006, pp. $83-150$

2. S. Schmuck, Design and Calibration of Thomson scattering polychromator for Wendelstein 7-X, this conference

3. H. Gross, Handbook of Optical systems, Wiley-VCH edited by Herbert Cross, Vol.1, Fundamental Optics, p 475-479, Vol. 3 Aberration Theory and Correction of Optical Systems pp. 549-551

4. J. M. Sasian, Image plane tilt in optical system, Optical engineering, 1992, Vol. 31(3), pp. 527-532

5. A. K. Forrest, Oblique imaging, magnification and image intensity, Mechanical Engineering Department, Imperial College of Science Technology and Medecine, London, Journal of Optics, A: Pure Applied Optics 1, 1999, pp. 697-701 